\title{
Mitigating dilemmas in postgraduate researcher mental health and well-being project implementation: critical reflections from three former implementers.
}

\author{
VALEIX, S., MOSS, R. and MORRIS, C.
}

This author accepted manuscript is deposited under a Creative Commons Attribution Non-commercial 4.0 International (CC BY-NC) licence. This means that anyone may distribute, adapt, and build upon the work for non-commercial purposes, subject to full attribution. If you wish to use this manuscript for commercial purposes, please contact permissions@emerald.com. 


\section{Studies in Graduate and Postdoctoral Edu}

\section{Mitigating Dilemmas in Postgraduate Researcher Mental Health and Wellbeing Project Implementation: Critical Reflections from Three Former Implementers}

\begin{tabular}{|r|l|}
\hline Journal: & Studies in Graduate and Postdoctoral Education \\
\hline Manuscript ID & SGPE-08-2020-0058.R1 \\
\hline Manuscript Type: & Main Section (Research and Theory Papers) \\
\hline Keywords: & $\begin{array}{l}\text { implementers, implementation, project, dilemmas, postgraduate } \\
\text { researcher, mental health }\end{array}$ \\
\hline \multicolumn{2}{|l}{} \\
\hline
\end{tabular}

\section{SCHOLARONE \\ Manuscripts}




\section{Structured abstract}

Methods:

Firstly, we recalled, listed and discussed our experiences of dilemmas as female project implementers of PGR-MHW projects and identified broad overarching themes. Secondly, we then each fleshed out one dilemma for the three themes according to the ones that carried meaning for how we personally experienced our role. Thirdly, we analysed what our accounts of dilemmas meant for project implementation and outcomes. We then linked findings to existing literature where similar tensions were identified, including how these could be mitigated.

Purpose:

This paper presents the critical reflections of three women implementers formerly working in projects that seek to support the mental health and wellbeing of postgraduate researchers, which has become a recent focus for UK researchers and policy makers. The paper offers an experiencebased perspective on tensions in PGR-MHW project implementation by providing personal accounts of several social dilemmas the authors encountered. From reflecting on experiences derived recommendations for mitigating such dilemmas when designing and delivering future projects.

Findings:

Dilemmas we experienced as implementers in PGR-MHW projects fit amongst three interconnected themes: Identity, Values and Motivations, and Relationships. We showed that, although they may be hard to see, the dilemmas presented in this paper impede project's success, outcomes for PGRs, and implementers' wellbeing. Mitigating such dilemmas when designing, funding, implementing, and evaluating future projects is not straight forward and our findings open avenues to tackle this problem.

Originality:

Focussing on reflections of female implementers, the paper provide an original perspective on PGRMHW project evaluation. Using reflective writing as a research tool allowed us to identify overlooked dilemmas in project implementation. Honest and critical accounts of implementers' experiences revealed important lessons such as different framings of project success, the intersection between the personal and the professional, and individual responsibilities in project networks.

Plain text summary:

People who work in projects that seek to support and improve the mental health and wellbeing of students (including doctoral researchers) encounter difficulties which are rarely discussed. In this paper, we have described situations where such difficulties arise, and thought about how to avoid them. We now know that these difficulties involve who the people are (their identity), why they are 
doing their job and what matters to them (their values/motivations), and how they interact with other people (their relationships). 


\title{
Mitigating Dilemmas in Postgraduate Researcher Mental Health and Wellbeing
}

\author{
Project Implementation:
}

\section{Critical Reflections from Three Former Implementers}

\section{Introduction}

The mental health and wellbeing (MHW) of Postgraduate Researchers (PGRs) has been a recent focus within the UK for researchers and policy makers, responding to recent evidence outlining areas of concern within the PGR population (Levecque et al., 2017; Vitae, 2018). PGRs report high levels of mental health difficulties (Evans et al, 2018; Dederfield et al., 2020; Hazel et al., 2020a; Hazel et al., 2020b). This, and recognition of limited understanding of the complexity of PGR-MHW calls for more quality research alongside supportive institutional interventions (Berry et al., 2020a).

Higher Education sector bodies, regulators and third sector organisations within the UK (Universities UK; Office for Students (OfS); Vitae) have sought to provide guidance and funding for projects that can support and improve PGR mental health and wellbeing (PGR-MHW projects)_in this context. A commissioned report released by the UK Researcher Development Organisation, Vitae, explored PGR-MHW (Metcalfe et al., 2018), finding that PGRs faced multiple challenges over the course of their studies with potential to impact negatively on mental health and wellbeing. Recommendations informed a Catalyst funding call from Research England and the Office for Students which supported 17 UK projects designed to support PGR-MHW (2018 - 2020). Project evaluations focussed on the impact of interventions for PGRs or those who support them on a continual basis (supervisors, student support services staff).

However, there is little data from staff who implement such projects on the ground - here called 'implementers' - and implementers' experiences and 
learning curves are often overlooked. The term implementer encompasses project team members who deliver project outputs, as these may have different official roles and hold different job titles across projects (researchers/ coordinators/ support service staff/ PGR project assistant, etc.). Here we differentiate implementers from others potentially involved in delivering project outputs (like academic leads) in that implementers do not have a non-project related full-time job. Similarly to higher education policy implementers in

8 Nienhusser $(2018,428)$ who "make sense of ambiguities that exist in policies while grappling with their personal beliefs and professional realities", PGRMHW project implementers are at the frontline of PGR worlds and therefore more able to see the gaps between initial intervention plans and actual impact. Down and Down $(2018,169)$, writing on sustainable development projects, says that "much of the literature focuses on ... the implementation process rather than on the actual implementers".

Here, we adapt and apply these insights to our investigation into implementers' experiences and dilemmas working on PGR-MHW projects.

Once funding ends, an important aspect of evaluation involves reflections from those at the project core, such as implementers (Williams et al., 2020; Shek et al., 2017; Washburn et al., 2018). Implementers have a unique overview of all strands and actors involved in the project, constituting valuable knowledge and expertise for planning future projects.

Such knowledge reveals tensions between project goals or policy-level based pathways and implementers' abilities, motivations, or behaviour, often translating as dilemmas for implementers. Drawing on the notion of 'dilemma' for understanding implementation (Bicchi, 2010), we understand this concept as used in Street-Level Bureaucracy literature (see Lipsky, [1980] 2010). Lipsky (2010) saw dilemmas as difficult choices civil servant implementers have to make when dealing with the public's needs which often translate as discretionary practices contradicting policies decided at higher levels. Revealing implementation dilemmas allows us to elucidate tensions between policy and practice (or between projects' needs/ directions and 
implementers' personal/ professional worlds and identities).

This paper offers a retrospective analysis of experiences from three former 'implementers' in PGR-MHW projects (the authors) with the following question as guiding focus: "What dilemmas have implementers of PGR-MHW projects faced, and, how could these dilemmas undermine project implementation and outcomes for PGRs?"

\section{Framework and Methods}

This paper draws on critical reflective research in which we examine three sets of personal experience of women working as implementers for government-funded PGR-wellbeing projects in contrasting UK universities. Reflective storytelling processes are drawn on in life history research in education (Goodson and Sikes 2001) to help make sense of interlinking personal and professional identities and experiences. Collective modes of storytelling have been deployed to capture academics' everyday experiences (Gannon et al. 2018; Author 3 et al., 2020; Res-sisters, 2017). Such methods offer a way into recognising and understanding lived complexities and capturing experiences which might not otherwise receive attention. They can make visible experiences, identities and emotional lives whose voices might otherwise go unheard due to academic hierarchies and unequal power relations (Author 3 et al., 2020), generating new insights for those engaged in PGR-wellbeing projects. It highlights that implementers often have relevant lived experiences to contribute and recognises the value of first-hand lived experience of researchers themselves (Grant, 2010).

Reflection can be understood as '...intellectual and affective activities in which individuals engage to explore their experiences in order to lead to new understandings and appreciation' (Boud et al, 1985, p. 3). Reflective practice and writing has been increasingly established across disciplines and professional spheres to create valuable learning opportunities and criticality (Bolton and Delderfield, 2018). Closely aligned to such practices, reflexivity is 
a valued component of social research which recognises research processes as embodied and situated (Mauthner and Doucet, 2003).

Reflective writing is characterised by 'self-reference and evaluative commentary' and often used for personal and professional development (Nesi, 2007). It is a recognized research method within professional and life history research (Fook, 2011; Plummer, 2001), adopted here as an innovative way of reflecting on past projects as 'people' not only defined by their role, but also by their social worlds. Reflective writing here works as a tool to capture sometimes invisible and intangible experiences like dilemmas, to which we then apply an analytical lens. It is also an ideal way to explore sensitive and personal dimensions of experience such as mental health which often go unspoken (Grant, 2010).

Adopting a thematic approach, analysis involved three stages: First, having written on and discussed experiences of dilemmas as project implementers, we identified broad overarching themes: Identity, Values and Motivations, and Relationships. Secondly, we each fleshed out one dilemma for the three themes identified in the first stage. Although each of us had experienced each type of dilemma presented here, the ones we selected and elaborated on carried meaning for how we personally experienced our implementer roles. Thirdly, we analysed what our accounts meant for our respective projects' implementation and outcomes. We then linked findings to existing literature where similar tensions were identified, including how these could be mitigated.

The themes we identified represent, from our experiences, significant dilemmas relevant to PGR-MHW project implementation. The Identity theme concerns the interaction and polarisation of operating as an academic implementer within an environment and research culture linked with and reported to exacerbate poor staff MHW (Guthrie et al., 2017; Wellcome Trust, 2020) while implementing interventions designed to support MHW. The joint themes of Values and Motivations concern tensions existing between projects 
1 and institutional imperatives and individual values like social justice that are

2 often key motivations for implementers to work in the field of MHW. The

3 Relationships theme explores the social structures and nature of interactions

4 in the teams where implementers operate, and how gaps within networks

5 may affect efficiency of project delivery and quality of project outcomes. Below

6 are three separate accounts, each addressing a key theme.

7 Findings: Dilemmas in PGR-MHW project implementation

8

Through the lens of reflective writing and drawing on examples of experiences of PGR-MHW project implementation, we show that there exist dilemmas in implementers' identities, values/motivations, and relationships. We also demonstrate how these dilemmas often contradict PGR-MHW projects' objectives, seriously impede outcomes for supporting better PGR-MHW, and undermine implementers' own wellbeing.

\section{Identity dilemmas (Author 3)}

I conducted research on a number of postgraduate- related projects and initiatives alongside doctoral studies: These included a Higher Education Academy (HEA) project (2007-2010) (Wisker et al., 2010) which investigated doctoral learning and identified aspects of experiences which impact on wellbeing (Author 3 et al, 2011). I simultaneously conducted institutional research and development on student MHW (Author 3, 2011); a subsequent HEA funded project specifically investigated links between PGR learning experiences and wellbeing (Author 3, 2011). This involved developmental activities and resource development. I was also involved in leading peer-to-peer initiatives as part of a scholarship programme at my institution.

My life at the time involved juggling multiple roles and identities as a doctoral researcher at one institution, a professional researcher working on multiple projects in another, and a lone mother of three. Even within individual projects, at any one time I would be acting as researcher, administrator, wellbeing event organiser, developer, facilitator and mental health advocate, speaking 
1 to a wide range of stakeholders and audiences including academic, internal 2 and external practitioners, students, and grassroots community workers. While this was an exciting and formative experience, it was challenging to manage everything. Identity dilemmas occurred in terms of where I 'fitted in': chiming with many PGR and early career experiences of non-belonging, I found myself caught between roles as student and researcher, student and staff (Author, 2020; Smith, 2020). Exacerbating non-belonging were experiences of devaluation of wellbeing work in academic culture, often positioned as not 'serious' academic work and viewed as an add-on rather than a necessity. There were assumptions that academics should not be involved in such work - student wellbeing viewed as an area for separate specialist services rather than a responsibility for all. As a 'wellbeing worker' (echoing Ahmed's (2012) concept of 'diversity workers'), I became a go-to person for students and staff to talk to about wellbeing issues. Due to high levels of commitment, I was happy to continually go above and beyond but this came at the risk of burnout. Significant emotional labour (Hochschild, 1983 ) is involved in hearing and responding to mental health issues and other serious problems and intervening where necessary. This led to dilemmas in terms of wanting to emotionally and intellectually invest in the project but also needing to maintain self-care and progress on my own $\mathrm{PhD}$ research.

These experiences were highly gendered. Emotional labour (Hochschild, 1983) involved in such wellbeing projects and pressures to undertake carework in the academy, is often one which falls to individual women (Morley, 1998). It is all-too-often invisibilised and under-valued (Lynch, 2010) and can affect women's career progress. Experiences as a lone parent while working as realities for many PGRs (Burford and Hook, 2020; Jazvac-Martek, 2009). There were inherent dilemmas involved in wanting to support others without becoming overloaded; between trying to meet project demands without overreaching, at the cost of my own academic goals, family life and health. 
My decision to disclose my history of mental health in the interests of de-

2 stigmatisation attracted mixed responses, from congratulations on being 'brave' to discomfort or pity, to assumptions about my abilities.

Comments about me being brave were double-edged - while meant well, they suggested underlying assumptions that really mental health challenges are something to be embarrassed about and should ideally be kept quiet. At times I felt myself labelled as the token 'person with mental health difficulties' on the project and seen as a spokesperson, rather than appraised for my research abilities; these experiences prompted subsequent dilemmas about disclosure. Lack of institutional support for my role meant that, as soon as the projects were completed with no immediate recourse to external funding, I had to find other work and took a long-term job as a student support worker on a lower pay grade with no promotion opportunities. This speaks to widespread precarity and short-termism in the university sector, associated with detrimental impacts for early career employees (UCU, 2019).

Alongside inhibiting academic and career development, job insecurity meant the potential for publication, dissemination and continued development of the project work was undermined. Precariousness undoubtedly contributed to periods of stress-related illness. My experiences suggest that the particular demands and complexities of wellbeing work need to be fully understood and valued. Especially, the valuable contributions of PGRs who already face an increasingly precarious academic climate should be recognised, as they can ironically end up 'bearing the brunt' of wellbeing initiatives intended to support them.

\section{Values and Motivations dilemmas (Author 2)}

Early in 2018, I had just received a pass for my PhD. Alongside the last year of my $\mathrm{PhD}$ (completed remotely from my host institution) and encompassing my viva, I had been working full, and later, part-time locally to support myself financially and for my CV. Of personal significance, at that time 
I also had first viewed the job advertisement for a Research Assistant attached to a Catalyst-funded PGR-MHW project (introduced earlier). When viewing this, I observed an instant and visceral reaction; internally I knew I 'had to do this', I had to apply for this post and hoped with every fibre of my body that I would get the opportunity to apply my skills to such a project. Why? I was motivated by my personal experience and that of my peers as PGRs, in addition to the value I placed on social justice and therefore, projects which aimed to benefit a diverse population particularly vulnerable to mental health concerns (Levecque, 2017). I will now discuss these motivations and values in greater depth.

Personally, the PhD process was one of great professional and personal challenge, alongside, at times, the enthralling sense of place within the academic community. Importantly, 'life' outside of the PhD happens at that same time. In my circumstances, this involved a variety of life events just before and over the course of my degree, including a number of house moves, the death of a friend, a family suicide, the start and end of various relationships, navigating my sexual orientation and identity, to name but a few. The life events I describe happened whilst I was hundreds of miles away from my familial support network. My peers also experienced numerous life events whilst undertaking doctoral study. Needless to say, my personal circumstances, combined with navigating an uncertain and pressured academic environment (particularly navigating academic precarity, noted by Author 3), impacted on my wellbeing and overall mental health. I sought support where I could - from mentors, friends, and the student counselling service.

Consequently, by the time I viewed the job advertisement for PGR Wellbeing, 'I knew' what it could feel like to be in a vulnerable position and in need of further support; recognising the need to equip individuals with information and mental health literacy, combined with adjusting the academic environment to create a more overall supportive institutional structure surrounding PGRs. I value(d) supporting others and many of my personal values could be considered 'other-orientated' (Korsgaard et al., 1996; Tassell and Flett, 2011). 
My PGR experience provided insight and became a motivating factor for pursuing the post and further projects. Moreover, I realised over the course of myPhD that I wished to apply my broad skillset (that I felt and feel fortunate for) into a career which benefitted others. If I was to pursue a research career, I wished to be involved in and lead projects that were applied and sought to immediately support vulnerable populations. The fact that I could be paid a salary in order to do so was a necessary, yet pleasant addition. According to self-determination theory (Deci and Ryan, 2000), such a desire could be described as an identified motivation; I wished to be involved in work that I perceived to have value and importance, with the secondary motivating factor being external (salary). Similar identified motivations have been reported in healthcare workers (Tassell and Flett, 2011). My motivation underpinned the value I placed on my work and involvement in PGR-MHW projects - to help others and contribute to something that could generate wider, systemic change, in line with a social justice framework (Tol, 2020; Powers and Faden, 2006). Thankfully, and much to my delight, I was successful in my application.

From a project needs and institutional imperative perspective (project team, senior management and external stakeholders), the project was seen as successful; we reached our pre-defined milestones and exceeded expectations, particularly in terms of outputs and dissemination. In contrast, from a personal point of view, I held and still hold mixed feelings about the academic indices of project success described previously. I recognise that I found great joy in working alongside and supporting PGRs to develop interventions, as well as providing and sharing space for PGRs to air their thoughts, ideas and concerns to different levels of stakeholders. It was selfaffirming to hear that the PGRs we had been working with had been passionate to engage in the project and had personally benefitted from it.

However, there were elements where project reach and scope were not as immediately widespread as I would have hoped. In a focus group, PGRs discussed how they felt that the project had not immediately influenced the institutional culture surrounding PGR mental health and overall support. 
1 Personally, even though I came to understand that culture change takes time,

2 I had been wondering whether my or the project's involvement had any impact and whether the project's activities were sustainable without funding. I had identified wholly with the project and was not only professionally but also personally invested in its outcome.

In addition to sustainability, I asked myself questions surrounding the blurred lines between tokenism, indicating minimal effort to facilitate the engagement of people (PGRs) in meaningful ways (Supple et al., 2015), and co-design and co-production with PGRs. PGRs were engaged in post-project design and suggested an abundance of ideas which, disappointingly, could not be incorporated within the project lifecycle. Such ideas included enhanced induction support for second and third year PGRs and further support for PGRs returning from field trips to combat isolation. From my perspective, their ideas were communicated passionately and clearly responded to identified needs within the PGR community. I sensed that it was empowering for PGRs to have an accessible forum to discuss their concerns and ideas.

But this sudden (temporary) empowerment and enthusiasm about the forum also reflected the absence of communication between PGRs and senior university staff before (and thus also likely after) the project. It was difficult to communicate to PGRs that some ideas could not be developed during the project, until a communication channel had been established by the 'appropriate' school (post-project), echoing Author 1's account below on dilemmas around relationships. Through a values/motivations lens though, this impossibility of adapting project plans to PGRs' needs was due to the 'limits of what could be achieved within the project lifecycle', an idea developed in the management sector (Labuschagne and Brent, 2005) and linked to managerial/ business-related values.

From my experience, definitions of project success differed between stakeholders in my project, based on the differences in values and 
1 considered a success from a project need and institutional imperative 2 perspective (focussing on outcomes, dissemination), I held my 'success' to be linked with the knowledge that my work had supported individual PGRs and that they found our interventions helpful (personal impact), as opposed to academic and project markers of esteem and managerial impact. In summary, reflecting upon my experiences has revealed values and motivation-based dilemmas existing between stakeholder and institutional perspectives.

Relationships dilemmas (Author 2)

Like Author 2, I worked on one of the Catalyst projects (as coordinator) and also entered the project with a strong personal interest in student mental health, having been a mental health advocate with disclosed lived experience of mental health difficulties as a PGR. Because I had studied science policy and professional integration around systemic challenges from a cultural anthropological perspective at this same university for the six years prior to that, I also saw this project through critical eyes with attention to the challenge of social interactions and network dynamics when managing complex problems, like student mental health.

The project relied on a wide range of stakeholders within (but also outside of) the university collaborating both through formal and informal channels, like the other Catalyst funded projects involving research and interventions on PGR-MHW. These stakeholders were academics, staff from different professional services, and PGRs from various schools and departments. Being the project's coordinator gave me a central and therefore ideal position to observe and understand the main dynamics in the project's network. However, the extent to which the project fitted in various staff's regular activities was not always clearly defined and kept changing over time. This meant that, through my eyes, the network operated in complex ways. Though complex networks' outcomes are hard to predict based on what we know at the start (Cardona-Meza and Olivar-Tost, 2017), there are central people who occupy key positions and who significantly influence the network's ability to satisfy its purpose, like gatekeepers (Pauget and Wald, 2013). 
In social network analysis, gatekeepers are 'nodes that form a connection

2 between disorganized others, or more specifically: actors in a network that 3 hold a position between other actors that are not linked directly' (Hulshof, 4 2012). Gatekeepers are a type of 'knowledge broker', whereby without them 5 information does not circulate between different groups of people (Burt, 1992; 6 Kirkels, and Duysters, 2010). In this project, gatekeepers were revealed to 7 be key actors to the network's delivery of outcomes and the creation of a 8 positive change. But if these individuals did not assume their role it meant that 9 key resources for the project's implementation could not be accessed and 10 used. Through the two accounts below, I illustrate how gatekeepers 11 influenced the network's efficiency for project delivery and therefore created 12 important relationship-based dilemmas relevant to the project's 13 implementation.

I myself acted as a gatekeeper between PGRs and the rest of the project team because of my closeness to the PhD process. First, I was still working on my own thesis corrections when I started working on the project, so officially still belonged to the student body. Second, I maintained friendships and cordial relationships with all the PGRs in my department alongside peer-to-peer connections in other departments. Third, when still a student, I had disclosed personal experience of depression and a general anxiety disorder during my PhD. Fourth, my positionality as a non-British female who looked younger than her age made me very approachable, I believe, to PGRs I met during the project. Thus, I was in a critical position to understand, reach and communicate to $P G R s$ around some of the project's strands like the PGR-led initiative scheme and PGR participation in our steering group.

However, this particular position in the network, which no one else occupied in my team, involved creating bonds with PGRs based on trust and accessibility. While PGR-staff relationships were perceived as lacking by 31 PGRs, 'trust' was underpinned by attending PGR-led events and being 32 available to answer the phone late at night or during the weekend - many 33 PGRs do not typically work nine-to-five Monday to Friday (Berry et al., 2020b; 
1 Metcalfe et al., 2018). 'Accessibility' meant that it was easy to talk to me about a recent funding cut (for overseas conference attendance, decided by university management) and to ask me if I could do anything about it. It also entailed PGRs asking me why there were no more counselling hours available and what 'my project' was doing about it. In both examples, for PGRs I was perceived as on 'the other' (university management) side, since I was staff. Also, as staff, I feared I might be seen as on 'the other' (PGRs) side as

8 if I would not understand the motivations behind keeping a low budget for PGR services/ support. This somehow left me feeling that I was trapped bound to fail each side - in a no-man's land. Simultaneously, it placed on me an underlying mediator-like responsibility for which support was critical.

In my years as a PGR and project coordinator, I heard many complaints about the difficulty of accessing timely help through counselling. Hence, one of our project's strands that involved 'work[ing] with local statutory NHS services to make it easierfor PGRs to access evidence-based psychological therapies' revealed important expectations for PGRs. Therefore, collaborating with the Counselling service team at our university (who was partnering with local NHS services) was critical for delivering this strand. A key person within the counselling services became our gatekeeper, linking us to the rest of the team and local NHS services. About a year into the project however, this person went on long-term leave and did not return before the end of the project. At this point, this person had just established working relationships with people in local NHS services to collaborate with us. It proved impossible for us to build new direct relationships with these key NHS people afterwards. Indeed, collaboration between professionals coming from different disciplines or sectors is a challenge Holmesland et al., 2010), which would have required time and resources we no longer had. Consequently, we lost the opportunity for collaboration and could not deliver this project strand so needed by PGRs.

\section{Discussion and Conclusion}


experiences of working on PGR-MHW projects have enabled us to make sense of complex experiences. As Dewey (1916) observed, an activity alone does not comprise experience but rather the meaning-making accorded to it through reflective processes. In line with our focus on dilemmas, Dewey (1933) suggests that moments of doubt, such as experiencing a dilemma, open up spaces from which new learning and professional insights can occur. In attending to these moments, our reflections offer valuable lessons for ourselves and also the wider sector in relation to wellbeing projects and experiences of PGRs.

PGRs, and especially those with family responsibilities, may find themselves struggling to manage multiple roles and identities (Jazvac-Martek, 2009). Equity related dimensions are vital considerations for wellbeingfocussed projects, attending to gendered, racialized and ableised aspects of undertaking wellbeing-focused academic and development work. Professional boundaries could be a vital aspect of training and project management on wellbeing projects, alongside robust infrastructures of support in teams and institutions. Attending to and addressing the widespread effects of increased academic precarity (UCU, 2019), including potentially detrimental MHW impacts, and ways in which they intersect with marginalised and disadvantaged PGR positionalities is paramount. 
Continued mental health awareness and anti-stigmatization training and activities in institutions could usefully challenge 'deficit' models of those who have or are experiencing issues and there are particular considerations for stakeholders in relation to the implications of and support for personal disclosures within the team. Explicit valuing of PGR voices and fostering inclusive PGR communities within institutions would go some way towards addressing feelings of non-belonging (Author 3, 2020). Participatory research frameworks could support this, ensuring better integration of postgraduate suggestions and sustainability of projects.

Considering the values and motivations dilemmas which Author 2 describes, definitions of project success can differ between stakeholders, involving interplay between project-based and more subjective values/motivations such as social justice. Given the importance social justice places on mental health and wellbeing (Powers and Faden, 2006), this values-based framework in the context of PGR-MHW projects should be considered, alongside 'traditional' indicators of academic esteem. It is important to create a continuous open and honest dialogue with PGRs during their period of study and to provide fora for seeking their opinions and ideas.

Moreover, PGRs need to be provided with sufficient access to different levels of stakeholders (including senior management) within their institution. The ability of PGRs to influence their experience is particularly important for PGR-MHW projects. Consequently, the creation of PGR-facing panels, similar to Patient and Public Involvement (PPI) panels (Fereday and Rezel, 2017), could be an accessible method for academics and takeholders alike to engage with PGRs in an open manner. Such panels may facilitate rapid engagement of PGRs in PGR-MHW projects that are being developed for internal/external funding calls, in order to ensure their relevance. Moreover, they could facilitate projects adopting a co-production approach to research (Slay and Stephens, 2013), whereby PGRs are involved at every stage from pre-design through to dissemination. Such considerations would strengthen impacts of PGRMHW projects seeking to consider project needs, alongside individual 
implementers' values and motivations.

Relationship dilemmas analysed through Author 1's experience suggest that PGR-MHW projects can involve complex networks of people operating in compartmentalised teams who collaborate in undefined ways and through informal channels. In these networks, central people like gatekeepers are key for collaboration to happen and project strands to be delivered. Therefore, mapping project networks and identifying gatekeepers from the start appears to be a good way to ensure collaborative relationships are created and maintained (Schröpfer et al., 2017; Lee et al., 2018). This could also include plans to identify and involve potential 'substitute' gatekeepers in order for them to step in if a primary gatekeeper has to leave within the project timeframe.

Equally, it is important to invest resources and paid time for implementers who are gatekeepers with PGR groups to build key relationships with PGRs, supporting them through belonging-related ambiguity and pressures. Since more women disclose mental health problems (Astbury, 2001) (which can facilitate gatekeeping as seen in Author 1's account earlier), and men find it easier to promote their interests as gatekeepers in networks (Van den Brink, 2014), and that caring and emotional labour in the academy often falls to women (Morley, 1998) we recommend that women implementers in MHW projects are provided with committed support to safely fulfil their role.

Although we separated dilemmas across three themes for the benefit of clarity and analysis, it appeared highlighted in our accounts that Identity, Values/Motivations, and Relationships dilemmas were interconnected. For instance, identity and relationship dilemmas reinforce each other around gatekeeping, trust and emotional labour that may be imposed on implementers, especially women. Values/motivation-based and identity dilemmas intersect when considering the personal aspects of being a PGRMHW project implementer. Author 3's account of identity dilemmas revealed many difficulties: juggling various personal and professional sometimes 
1 intertwined roles at the same time; feeling gendered burdens of responsibility

2 for emotional labour; struggling to fully belong in PGR/staff populations

3 (also highlighted in Author 1's account); wellbeing work being under-valued

4 and resourced and experiences of precarity, leading to inhibited academic

5 development. The identity theme encompasses a higher number of dilemmas

6 than in the other two themes (values/motivations and relationships). This may

7 indicate that the identity dilemmas are more prominent in PGR-MHW project

8 implementation. However, different implementers are likely to experience

9 projects and social dilemmas in significantly different ways and it cannot be

10 expected that implementers would relate to each story told in this paper.

11 Further, the number of dilemmas experienced while working on a project does

12 not necessarily correspond to the intensity with which dilemmas would affect

13 an implementer's work and wellbeing.

14

15

16

17

18

19

20

21

22

23

24

25

26

27

28

29

30

31

32

33

Since our identified implementation dilemmas are interconnected and may impact in different ways for different implementers, we consider that actions undertaken for mitigating dilemmas will encompass multiple dilemmas. In Table 1, we synthesise our recommendations to future PGR-MHW project implementers, managers, senior leaders, funders and evaluators to mitigate implementation dilemmas altogether.

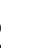

3

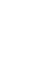

6


Table 1. Suggestions of actions to mitigate implementation dilemmas in future PGR-MHW projects across different stakeholders.

\begin{tabular}{|c|c|}
\hline Stakeholder & Mitigation \\
\hline PGR-MHW Project Implementers & $\begin{array}{l}\text {-Establish and discuss clear boundaries and risks to wellbeing when starting role and discuss with line managers as part of professional development } \\
\text { review processes } \\
\text { • Join groups that offer support re. precarious work (e.g. trade unions) }\end{array}$ \\
\hline PGRMH Project Managers & $\begin{array}{l}\text { - Co-create definitions of project goals and success with PGRs from the inception of and throughout the project } \\
\text {-Invest resources to support implementers who are gatekeepers working with PGR groups (especially women and early career employees/graduates) } \\
\text {-Identify gatekeepers and plan for substitute gatekeepers before project onset and revisit throughout project lifecycle } \\
\text {-Establish and discuss clear boundaries and risks to wellbeing when starting role with implementers } \\
\text { - Ensure implementers' wellbeing is addressed as part of research ethics approval processes } \\
\text { - Monitor wellbeing of the project research team in line with ethical practice and institutional policies, through regular communication and line } \\
\text { management meetings, making referrals to occupational health and putting reasonable adjustments in place as appropriate for those experiencing } \\
\text { mental health challenges } \\
\text { • Ensure any team members who publicly disclose mental health challenges receive guidance and support } \\
\text { - Build time for researchers to take up development opportunities in the research design and to work on publications and dissemination before funding } \\
\text { ends } \\
\text {-Strive for a research culture where mental health and wellbeing can be openly addressed and supported }\end{array}$ \\
\hline $\begin{array}{l}\text { Senior leaders (ex: university } \\
\text { managers/ executives) }\end{array}$ & $\begin{array}{l}\text {-Improve PGR access to senior leaders to talk about MHW (e.g. reverse mentoring) } \\
\text {-Address issues of precarity among postgraduate and early career researchers, attending to gender and ensuring equitable and dignified working } \\
\text { conditions - institutional initiatives such as Athena Swan could usefully focus more on early career experiences } \\
\text {-Ensure ethics panels consider whether staff wellbeing is being considered in ethics approval processes } \\
\text { - Creation of PGR-facing panels that sit with Graduate School(s), Faculties (appropriate forum - departmental/school, faculty, senior management } \\
\text { meetings) to inform policies and research which impact the population }\end{array}$ \\
\hline Funders & $\begin{array}{l}\text { - Consider as part of criteria for awarding funding to institutions } \\
\text { - Raise awareness of precarity and emotional labour (for women and/or PGR-early career implementers) with line managers/project leaders } \\
\text {-Provide clear guidance and support to line managers/project leaders so that they provide appropriate support to implementers before and } \\
\text { throughout the project } \\
\text {-Include accountability of managers' support to implementers in project evaluation }\end{array}$ \\
\hline Evaluators & $\begin{array}{l}\text {-Include the mental health and wellbeing of implementers themselves (qual+quant data) in evaluation plan } \\
\text {-Include assessment of managers' support and action for precarious staff and emotional labour to ensure their accountability }\end{array}$ \\
\hline
\end{tabular}


1 The field of mental health is one where implementers' are likely to closely identify with lived experiences of the beneficiaries. Therefore, this paper feeds into a wider literature relating to policy and practice in similar cases in other fields and involving non-university organisations. Further research could deploy longitudinal and/or quantitative methods to measure these dilemmas' impact on project implementation and implementers and to compare different types of dilemmas.

In conclusion, we advance that future PGR-MHW (possibly wider student $\mathrm{MHW}$ ) project implementers risk encountering some of the dilemmas described. These dilemmas being of a social nature may be more difficult to foresee than other factors influencing project implementation success like budget or organisation restructuring or natural disasters. That is why it was important to explore such dilemmas as potential barriers for project implementation through our stories. There is a need for more knowledge on how individual social worlds confront larger project's' directions in institutions like universities. This represents another reason why carrying out further research, using both qualitative and quantitative methods, would be beneficial.

Our findings indicate an ongoing need, beyond temporary projects, for broad culture change around mental health and wellbeing in universities. This is because, in practice, dilemmas undermine PGR-MHW project delivery as well as project implementers' wellbeing and health. Where responsibility for PGR-MHW is left to specific projects or individuals, measures are unlikely to be a success for everyone, be sustainable, or even happen at all.

When the world experiences an unprecedented pandemic due to Covid19, student MHW becomes even more of a challenge. Attending to potential implementation dilemmas in project design and delivery is key to shaping interventions and evaluating their impact. Examining and considering individual past experiences is necessary if we are to better design, deliver and evaluate future PGR-MHW projects in the UK and beyond. 


\section{References}

Astbury, J. (2001). "Gender disparities in mental health". In Mental health. Ministerial Round Tables, 54th World Health Assemble, World Health Organisation, Geneva, Switzerland.

Bicchi, F. (2010). "Dilemmas of implementation: EU democracy assistance in the Mediterranean:", Democratization Vol. 17 No. 5, pp. 976-996.

Boud, D., Keogh, R. and Walker, D. (1985). Reflection: Turning experience into learning, Kogan Page, London.

Burford, J. and Hook, G. (2019). "Curating care-full spaces: doctoral students negotiating study from home", Higher Education Research and Development, Vol. 38 No. 7 , pp. 1343-1355.

Burt, R. (1992). Structural holes: the social structure of competition. Boston, MA.

Cardona-Meza, L. S., \& Olivar-Tost, G. (2017). "Modeling and simulation of project management through the PMBOK® standard using complex networks", Complexity. 2017: 4791635.

Dewey, J. (1916). Democracy and education: an introduction to the philosophy of education, Macmillan, New York.

Dewey, J. (1933). How we think, DC Heath, Lexington.

Down, K. A. and Down, L. (2018). “Implementers' Perspectives on Creating Successful Education for Sustainable Development Projects". Caribbean Quarterly, Vol.64 No. 1, pp.167-187.

Fereday, S. and Rezel, K. (2017). Developing a patient and public involvement panel for quality improvement. https://www.hqip.org.uk/resource/developing-a-patient-andpublic-involvement-panel-for-quality-improvement/\#.XzEstkxFyUk (Retrieved August $8^{\text {th }}$ 2020).

Fook J. (2011). "Developing Critical Reflection as a Research Method”, in J. Higgs Jet al. (Ed,s) Creative Spaces for Qualitative Researching. Practice, Education, Work and Society, Vol 5. Sense Publishers, Boston. 
Gannon, S. et al. (2018). "On the Thresholds of Legitimacy: A Collaborative Exploration of Being and Becoming Academic", in Y. Taylor and K. Lahad. (Ed.s) (2018) Feeling Academic in the Neoliberal University: Feminist Flights, Fights and Failures, Palgrave Macmillan, New York.

Goodson, I. and P. Sikes. (2001). Life History Research in Educational Settings: Learning from Lives. Philadelphia: Open University Press, Philadelphia.

Grant, A. (2010). "Writing the reflexive self: an autoethnography of alcoholism and the impact of psychotherapy culture", Journal of Psychiatric and Mental Health Nursing, Vol. 17, pp. 577-582.

Guthrie, S., Lichten, C. A., Van Belle, J., Ball, S., Knack, A., and Hofman, J. (2017). "Understanding mental health in the research environment: A rapid evidence assessment", Rand health quarterly, Vol. 7 No. 3.

Hazell, C. M., Chapman, L., Valeix, S. F., Roberts, P., Niven, J. E. \& Berry, C. (2020a). Understanding the mental health of doctoral researchers: a mixed methods systematic review with meta-analysis and meta-synthesis. Systematic reviews, Vol. 9 No.1, pp. 1-30.

Hazell, C. M., Niven, J. E., Chapman, L., Roberts, P., Valeix, S. F., Cartwright-Hatton, S, \& Berry, C. (2020b). "Doctoral researchers experience greater depression and anxiety than working professionals". Submitted for publication to Nature Communications

Hoschschild, A. (1983) The Managed Heart: Commercialization of Human Feeling. University of California Press, California.

Holmesland, A.L., Seikkula, J., Nilsen, Ø., Hopfenbeck, M. and Arnkil, T.E. (2010). "Open Dialogues in social networks: professional identity and transdisciplinary collaboration", International journal of integrated care, Vol. 10.

Hulshof, L. P. (2012). Gatekeepers in Innovation Networks. Who are the gatekeepers in innovation networks of the German biotechnology sector? Masters thesis. 
Jazvac-Martek, M. (2009). "Oscillating role identities: The academic experiences of education doctoral students", Innovations in Education and Teaching International, Vol. 46 No. 3, pp. 253-264.

Korsgaard, M. A., Meglino, B. M., \& Lester, S. W. (1996). "The effect of other-oriented values on decision making: A test of propositions of a theory of concern for others in organizations", Organizational Behavior and Human Decision Processes, Vol. 68 No. 3, pp. 234-245.

Kirkels, Y. and Duysters, G. (2010). Brokerage in SME networks. Research Policy, Vol. 39 No. 3, pp.375-385.

Labuschagne, C. and Brent, A.C. (2005). Sustainable project life cycle management: the need to integrate life cycles in the manufacturing sector. International Journal of Project Management, Vol. 23 No.2, pp.159-168.

Lee, C.Y., Chong, H.Y., Liao, P.C. and Wang, X. (2018). "Critical review of social network analysis applications in complex project management", Journal of Management in Engineering, Vol. 34 No. 2, pp. 0401 - 7061.

Levecque, K., Anseel, F., De Beuckelaer, A., Van der Heyden, J., \& Gisle, L. (2017). "Work organization and mental health problems in PhD students". Research Policy, Vol. 46 No. 4, pp. 868-879.

Lipsky, M. (2010). Street-level bureaucracy: Dilemmas of the individual in public service, Russell Sage Foundation.

Lynch, K. (2010). "Carelessness: A hidden doxa of higher education", Arts and Humanities in Higher Education, Vol. 9 No. 1, pp. 54-67.

Mauthner, N. and Doucet, A. (2003). "Reflexive accounts and accounts of reflexivity in qualitative data analysis", Sociology, Vol. 37, pp. 413-31.

Metcalfe, J., Wilson, S., \& Levecque, K. (2018). “Exploring wellbeing and mental health and associated support services for postgraduate researchers",(May), 43. 
Morley, L. (1998) "All you need is love: feminist pedagogy for empowerment and emotional labour in the academy", International Journal of Inclusive Education, Vol. 2 No.1, pp. 15-27.

Author 3 (2011). "Open Minds: Towards a 'mentally well' university”, in (Marshall, L. and Author 3 (Ed.s) Taking Wellbeing Forward in Higher Education: Reflections on Theory and Practice, University of Brighton Press, Brighton.

Author 3 (2020). “Peering through the window looking in': Postgraduate experiences of non-belonging and belonging in relation to mental health and wellbeing", Submitted for publication.

Author et al. (2011). "Wellbeing and the Research Student" in Marshall, L. and Author 3 (Ed.s.) (2011). Taking Wellbeing Forward in Higher Education: Reflections on Theory and Practice, University of Brighton Press, Brighton.

Author 3 and Wisker, G. (2011). Troublesome Encounters: Strategies for managing the wellbeing of Postgraduate Education students during their learning processes. Escalate, Bristol.

Author 3 et al. (2020). Restorying imposter syndrome in the Early Career stage: reflections, recognitions and resistance, in Addison, M., Breeze, M. and Taylor, Y. (Ed.s.) Handbook of Imposter Syndrome in Higher Education. New York: Palgrave Macmillan. (Accepted for publication)

Author 2 et al, (2020) submitted for publication

Nesi, H. (2007). "The form, meaning and purpose of university level assessed reflective writing", In M. Edwardes (ed) Proceedings of the BAAL Annual Conference 2007. Scitsiugnil Press: London.

Nienhusser, H. K. (2018). "Higher education institutional agents as policy implementers: The case of policies that affect undocumented and DACAmented students." The Review of Higher Education, Vol. 41 No.3, pp. 423-453.

Pauget, B. and Wald, A. (2013). "Relational competence in complex temporary organizations: The case of a French hospital construction project network", International Journal of Project Management, Vol. 31 No. 2, pp. 200-211. 